\title{
Tecnologías de la Información y las Comunicaciones en la Prevención de la Obesidad
}

\author{
Information and Communication Technologies for the \\ Prevention of the Obesity
}

\author{
María Victoria Moreno Cano' \\ ${ }^{1}$ Fundación Séneca, Agencia de Ciencia y Tecnología. Región de Murcia, España \\ Fecha de recepción: 19/10/2016 - Fecha de aceptación: 28/01/2017
}

\begin{abstract}
Resumen
El número de personas con obesidad ha crecido a nivel mundial desde 105 millones de personas en 1975 hasta 640 en 2016, y esta cifra no para de aumentar. Si las tendencias actuales no cambian, un $20 \%$ de la población mundial será obesa para el año 2025. A pesar de que existen regiones en el mundo en las que la pobreza y la hambruna matan a decenas de miles de personas cada día, en los países desarrollados y emergentes el crecimiento económico ha traído consigo más y más comida, pero sobre todo, muchas calorías poco saludables y estilos de vida más sedentarios. Los gobiernos, incapaces de detener esta epidemia sanitaria, tendrán que prepararse para unos costes médicos cada vez mayores. Ante esta situación, es evidente la necesidad de proponer nuevas políticas que permitan frenar el aumento de la obesidad en la población, para así evitar que se produzca una epidemia de severa obesidad. En este artículo resaltaremos aquellas políticas que incluyen tecnologías que permitan asegurar la saludabilidad de los productos alimentarios que se ingieren, que propongan programas mejorados de entrenamiento para la salud, aumenten el nivel de autoeficacia, autorregulación y motivación para cambiar el comportamiento alimentario del usuario.

Palabras clave: Obesidad, Prevención, Tecnologías de la información y las comunicaciones, Aplicaciones móviles
\end{abstract}

\begin{abstract}
The number of people suffering obesity has grown at world level from 105 millions in 1975 until 640 in 2016, and this number is still increasing. If this trend does not change, it is estimated that the $20 \%$ of the world population will be obesity by 2025. Although there are several regions in the world where the poverty and the famine is killing to thousands of people every day, in the developed countries the economical growing has derived in more and more food, but above all, in a lot of unhealthy food and more sedentary lifestyles. The Goverments are not able to stop this health epidemic, so they have to be ready to cope with very high medical costs associated to this problem. Due to this situation, it is evident the requirement to propose new policy measures to stop the obesity in the population, and avoid an epidemic of severe and global obesity. In this paper it is described some of the technological measures whose aim is to monitor and ensure the good quality of food, and propose different training programs for the health, increasing their effectiveness through the self-regulation and the encourage of people to change their nutritional behaviour.

Keywords: Obesity, Prevention, Information and communication technologies, Mobile application
\end{abstract}

Correspondencia: victoria.moreno@coit.es

Rev Esp Comun Salud 2017, 8(1), $107-113$ https://doi.org/10.20318/recs.2017.3610 Página 107 


\section{Introducción}

La obesidad constituye un peligro para la salud pública que afecta a todos los grupos etarios y socioeconómicos, afectando tanto a países desarrollados como en vías de desarrollo. Este problema ya ha sido reconocido como un factor de riesgo para enfermedades crónicas importantes como la presión arterial alta, enfermedades cardiovasculares, diabetes tipo II y algunos tipos de cáncer (riñón, tiroides y páncreas), causando un alto impacto en el gasto sanitario y millones de muertes al año en todo el mundo. Sin embargo, y a pesar de constituir uno de los desafíos más serios de este siglo para la salud pública, sus principales determinantes no han sido completamente clarificados todavía.

Athina Raftopoulou, una investigadora griega del programa de doctorado de Economía de la Universidad de Barcelona, trabaja en una tesis para analizar los factores que provocan la obesidad dentro de un entorno socioeconómico, y sobre políticas útiles de prevención para su disminución. Este proyecto ha recibido financiación a través de la Crowdfunding Campaign para su puesta en marcha en colaboración con la Universidad de Barcelona ("UBEconomics | Raftopoulou, Athina", 2016). Según dijo la investigadora en una entrevista que le realizaron, "La obesidad no es un problema individual sino social, ya que acarrea un gran coste a la salud pública y reduce la productividad".

Los malos hábitos alimentarios en Europa se han convertido en un gran problema de salud. De hecho, son un importante factor de riesgo para el desarrollo de la obesidad y de los trastornos alimentarios que causan un grave perjuicio entre la población. El número de personas obesas se ha triplicado en Europa en los últimos 20 años y España es el segundo país del continente con la mayor tasa de obesidad en menores, que ya alcanza al $28,3 \%$ de la población infantil.

En la población infantil se viene observando además que los hábitos de tiempo libre de los niños/as ha cambiado mucho en los últimos años debido al desarrollo tecnológico. Aunque estos cambios presentan ciertas ventajas, también tienen sus inconvenientes pues están propiciando la aparición del sedentarismo. Por tanto, la sobrealimentación y el sedentarismo son factores que influyen en el peso corporal de las personas provocando obesidad (Alcaraz, 2000).

En este contexto, las tecnologías de la información y las comunicaciones (TIC) pueden ser de gran utilidad para ayudar a combatir el gran problema de la obesidad, sobre todo la infantil. Su impresionante ritmo de crecimiento e implementación en los últimos años ha facilitado la práctica clínica en los servicios de salud, incluida atención primaria.

Actualmente existen numerosas investigaciones que analizan cómo la tecnología puede ayudar en el diagnóstico, prevención y tratamiento de esta enfermedad. Entre estos avances tecnológicos se encuentra la realidad virtual, Internet, los teléfonos inteligentes, etc. Todas estas tecnologías se han 
usado de diferentes maneras, entre ellas en psico-educación, auto-ayuda, tratamientos, foros de apoyo, consultas médicas, monitorización de síntomas y cuidados posteriores.

En este artículo se realiza una revisión del estado global de la obesidad basada en el mayor análisis realizado hasta la fecha sobre esta enfermedad (Finucane et al., 2011), y se plantean algunas de las soluciones propuestas, destacándose aquellas basadas en la aplicación de las TIC.

\section{El mayor análisis global sobre obesidad}

Majid Ezzati, profesor de la Escuela de Salud Pública del Imperial College en Londres, llevó a cabo un estudio sobre obesidad en el que involucró a más de 700 investigadores de todo el mundo (Finucane, 2011). Estos investigadores analizaron los datos de peso y altura de casi 20 millones de adultos en 186 países. Los resultados de este estudio fueron publicados en la revista "The Lancet".

El Índice de Masa Corporal (IMC) se calcula dividiendo el peso de una persona en kilos, por la altura en metros al cuadrado, y sirve para indicar si el peso es saludable o no.

Así, cuando el IMC supera los 25 puntos puede hablarse de sobrepeso, obesidad si es mayor de 30 y obesidad mórbida cuando supera los 40 puntos. Ezzati y su equipo analizaron cómo en las últimas cuatro décadas el IMC medio en hombres pasó del 21.7 al 24.2, mientras que en mujeres pasó del 22.1 al 24.4. Un aumento que equivaldría a un incremeto de peso de 1.5 kilos de media cada diez años, según reconocen los investigadores que participaron en el estudio. De hecho, sus predicciones apuntan que si continúa esta tendencia, el $18 \%$ de los hombres y el $21 \%$ de las mujeres serán obesos en el año 2025.

El estudio realizado reveló, además, cómo la población adulta de Estados Unidos es la que presenta el mayor IMC del mundo, mientras que la de Japón es la que tiene los niveles más bajos entre los países desarrollados. Asimismo, en Estados Unidos y en China es donde viven más hombres y mujeres con obesidad. En Europa, los IMC más bajos se encontraban en las mujeres de Suiza y en los hombres de Bosnia.

La obesidad mórbida - cuando el peso de una persona puede llegar a interferir con algunas funciones físicas básicas como la respiración y caminar - afecta ya a alrededor de un $1 \%$ de hombres y $2 \%$ de las mujeres. En total, 55 millones de personas en el mundo son obesos mórbidos.

España se encuentra entre los países de la Unión Europea donde el impacto de la obesidad sobre la mortalidad evitable es uno de los más altos, aproximadamente el responsable del $5.5 \%$ del total. Además, la epidemia de la obesidad en España se caracteriza por su diversa población y la desigualdad de ingresos del país, convirtiéndolo en un ambiente prospero para que se realicen importantes investigaciones. Además en España, el Ministerio de Salud gasta 1.2 billones de euros por año en enfermedades relacionadas con la obesidad. 
Majid Ezzati señaló en su estudio que la reducción de la tasa de personas que no consiguen suficientes calorías en los países en desarrollo está directamente relacionada con el crecimiento de la obesidad en esos mismos países. "El asunto realmente se puede reducir a que hay personas que no tienen acceso a suficiente comida, y personas que no tienen acceso a suficiente comida saludable", explica Ezzati.

La explicación es simple: el precio de la fruta, de los vegetales frescos o de los productos integrales es notablemente más alto que el precio de los carbohidratos refinados.

La obesidad en el mundo en desarrollo no es una sorpresa para la FAO - Organización de las Naciones Unidas para la Alimentación y la Agricultura - "Ya sabíamos que el mundo producía suficientes alimentos para todos -afirma Barbara Burlingame, Oficial Superior del Grupo de Estimación y Evaluación de las Repercusiones de la Nutrición de la FAO-. Desafortunadamente, los alimentos no siempre llegan a quienes más los necesitan". Por tanto, el hambre es una consecuencia, y la obesidad otra.

Además, prácticamente todas las personas que padecen hambre y muchos de los que tienen sobrepeso sufren debilidad por otro tipo de malnutrición: la falta de vitaminas y minerales, conocida como deficiencia de micronutrientes. "Solía pensarse que si las personas obtenían suficiente energía de su alimentación, los micronutrientes vendrían por añadidura -afirma la Dra. Burlingame- Pero las personas cada vez ingieren más alimentos de mala calidad, que llenan el estómago pero siguen dejando el organismo sin los micronutrientes necesarios".

Si bien la información sobre la obesidad en los países en desarrollo es limitada, los estudios preliminares indican que algunas de las mismas deficiencias de micronutrientes que padecen las personas subalimentadas, las sufren también los sobrealimentados. Dos de las más comunes son la anemia, por falta de hierro, y la deficiencia de vitamina A, causa frecuente de ceguera entre niños menores de cinco años de edad.

En algunos países desarrollados se están empezando a implantar tasas a alimentos que fomentan la obesidad como las bebidas azucaradas, pero el esfuerzo es claramente insuficiente. Mientras, los países en desarrollo parece que se enfrentan a una nueva consecuencia más de su posición en el teatro mundial, con sistemas sanitarios deficientes y pocas alternativas para alimentar a su población, tienen menos armas que nadie para luchar contra la epidemia sanitaria.

\section{Algunas soluciones}

Para combatir el problema de la obesidad se proponen adoptar medidas globales y coordinadas que incluyan, entre otras cuestiones, luchar contra la subida de precios de los alimentos más saludables o las diferencias que hay en comparación con otros menos saludables, o fijar impuestos para los productos procesados o con alto nivel de azúcar. 
La FAO mantiene que un acertado planteamiento de la nutrición debe orientarse a la calidad así como a la cantidad. Una de las misiones más importantes es promover una alimentación diversificada, que contenga los alimentos tradicionales, por lo general bien equilibrados y muy nutritivos.

Las autoridades políticas deberían implementar políticas específicas, tales como el desarrollo de entornos que promuevan y apoyen una vida activa, aumentar las oportunidades para la realización de actividades físicas en lugares públicos, implementar estrategias de prevención y ofrecer incentivos a los mercados existentes para ofrecer una alimentación más sana.

En este contexto, las TIC pueden constituir una herramienta en la percepción del riesgo, búsqueda de apoyo social y auto-cuidado en personas con obesidad. En primer lugar, las TIC pueden ofrecer herramientas de diagnósis eficaces y sencillas que permitan identificar tanto a la población con obesidad como a la población en riesgo. Dado que en muchos casos, al inicio del transtorno no hay conciencia de enfermedad, la demanda no siempre estará claramente definida, siendo necesario interpretar determinados síntomas, como subidas o bajadas repentinas de peso, fracasos en programas de pérdida de peso, patrones de alimentación desorganizados, etc. Las evaluaciones sistematizadas a través de Internet y con ayuda de dispositivos móviles pueden ser de gran ayuda para el clínico. En el siguiente apartado se analiza con más detalle los beneficios que se pueden conseguir con la aplicación de las TIC en la prevención de la obesidad.

\section{Aplicaciones TIC para combatir la obesidad}

Con la evolución de la tecnología se está produciendo una verdadera revolución en la medicina con la irrupción de dispositivos dotados de un enorme potencial comunicativo. En este sentido, el teléfono móvil y el entorno de las aplicaciones móviles ofrecen un inmenso abanico de posibilidades para el mundo de la salud que se concreta en las llamadas aplicaciones $\mathrm{mHealth}$.

mHealth es aquella parte de la eHealth (salud electrónica/e-salud) que la Organización Mundial de la Salud (OMS) la define como "mHealth es la práctica de la medicina y la salud pública soportada por dispositivos móviles como teléfonos móviles, dispositivos de monitorización de pacientes, asistentes personales digitales y otros dispositivos inalámbricos."

Los teléfonos inteligentes (smartphones) pueden ofrecer herramientas muy útiles para el control de sintomatología y el seguimiento de las prescripciones clínicas. Existen aplicaciones que permiten el registro y supervisión del estado de ánimo, pensamientos disfuncionales, ingesta, actividad física, etc. (Oliver et al., 2013). Todas estas aplicaciones pueden ayudar a los pacientes a implementar, en la vida cotidiana, muchas de las prescripciones realizadas por el clínico, a la vez que permite al médico llevar una supervisión en tiempo real de la progresión del paciente.

Entre las ventajas del uso de las TIC destaca su facilidad para acceder a gran número de pacientes y personas en riesgo a un coste relativamente bajo, sin problemas de horario y a gran velocidad (Bauer 
\& Moessner, 2013). Otra ventaja es la posible disminución de la resistencia de los pacientes a buscar ayuda profesional. El anonimato que Internet ofrece les permite revelar información que, en otro tipo de circunstancias, pudiera ser más difícil de conseguir. Además, tanto Internet como las aplicaciones móviles pueden ofrecer una atmósfera de bienestar, seguridad y fácil acceso para proporcionar educación e información útil.

Como ejemplos de aplicaciones móviles aplicadas a combatir la obesidad, a continuación citamos dos de las mencionadas en el "informe que recoge las 50 mejores apps de salud en español" ("Informe 50 mejores APPs de salud en español", 2014):

- Endomondo. Aplicación de carácter social en la que el usuario, registrándose a través de Facebook, accede a un entrenador personal y puede compartir con el resto de usuarios ejercicios, rutas, etc. Incorpora GPS y medición del ritmo cardíaco.

- iDoctus. Ayuda a la práctica clínica del médico e incluye servicios de documentación, bases de datos de medicamentos actualizadas, archivo multimedia y apoyo al diagnóstico. Ganadora en SeedRocket 2011 y finalista en Ideas Sanitas 2013.

Obviamente las TIC también presentan problemas y limitaciones, tales como consideraciones éticas (privacidad y condencialidad, uso de conexiones seguras, etc.), dificultades para la comunicación interpersonal (problemas de empatía, sensación de cercanía, etc.), problemas de incumplimiento y abandono, entre otros.

Son escasos los estudios controlados aleatorizados sobre la obesidad en atención primaria que evalúen la eficacia y el coste-efectividad de los tratamientos cuando se incluyen TIC. Los expertos coinciden en que es pronto para confirmar su eficacia, ya que se desconoce el perfil de paciente que se benefecia de este tipo de programas, el grado de apoyo necesario (virtual, presencial, telefónico, e-mail), y el nivel de abandono. Pese a estas limitaciones, las TIC en atención primaria podrían constituir una herramienta adecuada para mejorar el diagnóstico, la prevención y el tratamiento de la obesidad, ofreciendo un gran potencial para reducir las barreras a la búsqueda de ayuda, facilitar el acceso al tratamiento y contribuir a una optimización general de la atención a la población.

Por este motivo, actualmente se está invirtiendo en proyectos para aplicar las TIC en la prevencion de enfermedades relacionadas con transtornos alimentarios. Uno de estos proyectos es MEAL (Modifying Eating Attitudes and Actions through Learning) (Research, 2016), en el que participan grupos de investigación de la Universidad Jaume I de Castellón (UJI) y la Universidad de Valencia como expertos en desórdenes psicológicos y terapias de tratamiento de la obesidad infantial. El proyecto MEAL ofrecerá, a través de una herramienta TIC, una metodología de formación para los profesores, estudiantes y profesionales de la nutrición que les permitirá adquirir, entrenar y ofrecer a los niños educación nutricional básica y entrenamiento en conductas alimentarias saludables, según añaden las mismas fuentes. 


\section{Referencias bibliográficas}

Alcaraz Portero, E. (2000). La alimentación y el sueño. Bases para un buen desarrollo de los hijos. Madrid: Editorial EOS.

Bauer, S., \& Moessner, M. (2013). Harnessing the power of technology for the treatment and prevention of eating disorders. International Journal of Eating Disorders, 46(5), 508-515.

Finucane, M. M., Stevens, G. A., Cowan, M. J., Danaei, G., Lin, J. K., Paciorek, C. J., \& Farzadfar, F. (2011). National, regional, and global trends in body-mass index since 1980: systematic analysis of health examination surveys and epidemiological studies with 960 country-years and 9. 1 million participants. The Lancet, 377(9765), 557-567.

Informe 50 mejores APPs de salud en español. (2014). http://www.theappdate.es/static/media/uploads/2014/03/Informe-TAD-50-Mejores-Apps-deSalud.pdf

Oliver, E., Baños, R. M., Cebolla, A., Lurbe, E., Álvarez-Pitti, J., \& Botella, C. (2013). An electronic system (PDA) to record dietary and physical activity in obese adolescents. Data about efficiency and feasibility. Nutr Hosp, 28(6), 1860-1866.

Research, F. (2016). MEAL project. Mealproject.eu. Consultado el día 2 de Agosto de 2016 de la World Wide Web: http://www.mealproject.eu/

Raftopoulou, Athina. (2016). UBEconomics. Consultado el día 2 de Agosto de 2016 de la World Wide Web: http://www.ub.edu/ubeconomics/phd_students/raftopoulou-athina/

\section{Agradecimientos}

Este trabajo ha sido financiado por la Fundación Séneca-Agencia de Ciencia y Tecnología de la Región de Murcia, por medio de una ayuda del Programa de "Talento Investigador y su Empleabilidad" en la modalidad de becas de formación posdoctoral (Consejería de Educación y Universidades de la Región de Murcia). 